\title{
A rapid literature review on inequalities and ethnicity in healthcare workers' experiences of delivering care during the COVID-19 pandemic
}

\author{
Georgia Chisnall and Cecilia Vindrola-Padros
}

\author{
Georgia Chisnall (Corresponding Author) \\ Affiliation: Rapid Research Evaluation and Appraisal Lab (RREAL), University College London, \\ London \\ Email address: georgia.chisnall.19@ucl.ac.uk \\ ORCID: https://orcid.org/0000-0002-1524-4564। \\ Cecilia Vindrola-Padros \\ Affiliation: Rapid Research Evaluation and Appraisal Lab (RREAL), Department of Targeted \\ Intervention, University College London \\ Email address: c.vindrola@ucl.ac.uk \\ ORCID: https://orcid.org/0000-0001-7859-1646
}

\begin{abstract}
A rapid literature review was conducted regarding inequalities faced by frontline BAME healthcare workers (HCWs) responding to the COVID-19 pandemic within the United Kingdom. A number of key themes and sub-themes were identified within the literature. Firstly, in relation to viral exposure.

BAME HCWs are more likely to be exposed to COVID-19 on account of: frontline prevalence (driven by pre-existing systemic racism pre-pandemic, disproportionate redeployment and location of redeployment compared to White counterparts); complications with Personal Protective Equipment (PPE) (driven by lack of access, training and fit-testing compared to White counterparts); and, being afraid to speak up in a 'bully' culture (redeployment and PPE access worse for minoritized groups due to lack of ability to say 'no', fearful of repercussions). Secondly, in relation to emotional impact. BAME $\mathrm{HCW}$ may experience heightened and unique emotional strain on account of: increased anxiety (due to their increased mortality risk, a greater 'tear' between personal risk and sense of duty, and through risk posed to family due to intergenerational living); and, due to racial abuse (experience of racially motivated verbal abuse from patients or patient families, and in some instances even peers, can dramatically change experience of providing care). There is a clear wealth of evidence which exemplifies inequalities in BAME HCWs' experiences of care provision during COVID-19. Steps should be taken not only to prioritise physical BAME HCWs' wellbeing but also their emotional and mental health needs. There is greater need for primary research, in particular qualitative data to narrate and capture the lived experiences of BAME HCWs during this pandemic.
\end{abstract}

Declaration of interest: none

Author's contributions: GC conducted the rapid literature review and drafted the manuscript with review and editing from CVP

Keywords: BAME, healthcare worker, COVID-19, inequality

Current categorisations of ethnic populations are imprecise, time and context dependent and do not translate globally despite sharing international relevance (Selvarajah et al., 2020). When using categorisations, such as Black, Asian and Minority Ethnic (BAME) in the United Kingdom (UK) or Black, Indigenous and People of Colour (BIPOC) In the United States (US), it is important to be mindful of the historic and geographical contexts which have given rise to these categorisations (Selvarajah et al., 2020). These contexts include connections to suppression, for example, being used to categorise immigrants in the 1940s and 1950s (Selvarajah et al., 2020). The issues with such categorisations, however, go far beyond historical context (Selvarajah et al., 2020; Abbas, Memon, Khattab, \& Abbas, 2020). Notably, these terms present multiple ethnic groups as homogeneous, overlooking critical social and cultural differences between groups; this also results in overlooking uneven power structures between these groups and variation in systemic exclusion and challenges between them. While categorisation can be helpful in identifying disproportional health burdens, inappropriate use not only presents significant challenges to data analysis and policy making but also endorses the social clustering as 'valid' which is problematic. Instead, more accurate categories which acknowledge the complexity of ethnicity which avoid merging diverse groups and regions are needed. While this report uses the term BAME this should not be seen as support for, or validation of, the use of this categorisation, rather this reflects the categorisation used by many of the articles the review findings have been based upon which is intertwined with data outputs. 


\section{A rapid literature review on inequalities and ethnicity in healthcare workers' experiences of delivering care during the COVID-19 pandemic}

\section{SETTING THE SCENE}

\section{Disproportionate COVID-19 disease and death in BAME populations}

By April 2020 it became apparent that the burden of disease and death from COVID-19 was overrepresented within BAME populations, which triggered a number of investigations into the phenomenon (Farah \& Saddler, 2020). The whistle blowing data in the UK was produced by the Intensive Care National Audit and Research Centre (2020), which revealed that $34 \%$ of COVID-19 patients in critical care wards were from BAME backgrounds, despite only constituting $17 \%$ of the UK population. This was shortly followed by a study from the Office for National Statistics (ONS, 2020) which found that after accounting for age, Black males and females were respectively 4.2 and 4.3 times more likely to die from COVID-19 than their White ethnic counterparts. This finding was also statistically significant for those of Bangladeshi, Pakistani, Indian, and Mixed ethnicity backgrounds. Even after accounting for socio-demographic factors and other data captured by the 2011 Census, the likelihood of death for men or women of Black ethnicity was still 1.9 times greater than those of White ethnicity. Similar inflated risk was observed for those of Bangladeshi and Pakistani ethnicity with values of 1.8 and 1.6 for males and females respectively. This was deemed notable, as while differences between ethnic groups in COVID-19 mortality could partially be accounted for through preexisting socio-demographic factors such as economic disadvantage, a notable proportion of the difference remained unaccounted for.

This was further compounded by a joint study from the University of Oxford and the London School of Hygiene and Tropical Medicine which also found that the observed disparity between nonWhite and White ethnic groups was only partially attributable to pre-existing clinical factors or deprivation (Williamson et al., 2020). A study by University College London also ruled out that this was due to geographical region finding that after accounting for this and age that Black and Minority Ethnic (BME) groups were still two to three times more likely to die from COVID-19 (Aldridge et al., 2020). Many found it concerning that the observed disparity could not be accounted for and growing media coverage prompted the government to commission Public Health England (PHE) to conduct a rapid review of disproportionate impact of COVID-19 (Farah \& Saddler, 2020). The report detailed that the highest-diagnosis rates were in Black ethnic groups, with the lowest in White ethnic groups. Survival rates were also implicated - individuals from Chinese, Indian, Pakistani and other Asian, Caribbean and other Black ethnicities had between 10-50\% higher risk of mortality compared to White British ethnicities. A follow-up report acknowledged that racism, discrimination and social inequality may have contributed to the death rates observed in minority ethnic groups (lacobucci, 2020). This review seeks to explore this inequality through the lens of healthcare workers (HCWs) providing care on the frontline during the COVID-19 pandemic.

\section{BAME populations in the healthcare workforce}

A sizable $20 \%$ of the 1.2 million staff employed by the National Health Service (NHS) are of BAME background (NHS Workforce Race Equality Standard; NHS WRES, 2019). As observed in the wider public, BAME individuals significantly over-represent healthcare worker COVID-19 deaths. This issue was initially exemplified by the first 11 doctors to die in the UK all belonging to BAME backgrounds (Otu, Ahinkorah, Ameyaw, Seidu, \& Yaya, 2020). An initial analysis of the first 106 confirmed COVID$19 \mathrm{HCW}$ deaths reported by April 22 revealed that $63 \%$ belonged to BAME backgrounds despite only representing $20 \%$ of the workforce (Cook, Kursumovic, \& Lennane, 2020). More specifically, while BAME account for approximately $20 \%$ of nursing and support staff and $44 \%$ of medical staff, they respectively account for $64 \%$ and $94 \%$ of deaths in these working groups. Furthermore, that more than half of health and social care deaths represented individuals born outside of the UK despite 
representing only $18 \%$ of NHS staff. Interestingly, high-risk specialities such as anaesthesia and intensive care were under-represented.

As in the broader public, it is not clear what drives the unaccounted proportions of this disparity. Over the following months, a number of studies, commentaries, and reputable journal news articles have been published, which shed some light on, not only the factors which may drive this disparity, but also how this creates inequalities in experiences between HCWs providing frontline care during the COVID-19 pandemic. This report seeks to summarise key themes in the relevant literature following a thematic and narrative synthesis of articles identified from an in-depth literature review.

\section{KEY FACTORS IN BAME HCW INEQUALITY}

\section{Viral exposure: BAME HCWs are more likely to be exposed to COVID-19}

a. Frontline prevalence: BAME HCWs are more likely to be exposed to the virus, and hence subsequent infection, disease and death due to their frontline prevalence. The identified literature considers pre-existing systemic racism pre-pandemic, disproportionate redeployment and location of redeployment in regard to this issue.

The aforementioned PHE review acknowledged the increased risk of exposure of BAME HCWs to COVID-19 due to occupation of front-line positions (PHE, 2020). This has also been identified in a cross-sectional survey of BAME HCWs $(n=200)$ : 'it is evident that the clear majority of the BAME healthcare workers are on the front line in their routine work, therefore they are at higher risk of contracting COVID-19' (Moorthy \& Sankar, 2020, p.490). Otu, Ahinkorah, Ameyaw and Seidu (2020) also point-out that BAME communities are more likely to reside in larger UK cities such as London, Birmingham and Manchester where COVID-19 rates are highest resulting in densely populated, highrisk wards.

It has been suggested the over-representation in frontline roles may be due to pre-existing base job roles rather than disparities in re-deployment (Chaudhry, Raza, Raja \& Ahmad, 2020). Prepandemic, it was acknowledged that BAME staff, nurses in particular, were more likely to be in patient contact professions than their white counterparts (NHS WRES, 2019). This, however, would still reflect systemic racism as, in part, the reason why minority ethnic groups are over-represented at lower NHS grades is because White applicants are 1.46 times more likely to be successful in being appointed than those of ethnic minority backgrounds - the rate of which is even higher in acute trusts which are the centre of COVID-19 care provision (NHS WRES, 2019). Furthermore, BME staff are 1.5 times more likely to enter a formal disciplinary process than their White counterparts (NHS WRES, 2019). In stark terms, as pay band increases from Band 5 to senior manager level the proportion of BME staff decreases from $24.5 \%$ to $6.5 \%$ (NHS WRES, 2019).

Having said this, disparities in redeployment cannot be ruled out as a pervasive BAME HCW experience. As reported in Ford (2020), Carol Cooper, the head of equality, diversity and human rights at Birmingham Community Healthcare NHS Trust, reported that BAME nurses and healthcare assistants felt they were being selected to work on COVID-19 wards more than white colleagues. In a qualitative study on HCWs led by RREAL, we also found that women were more likely to take on these roles due to their overrepresentation in the nursing workforce (Regenold \& Vindrola-Padros 2021).

b. Complications with Personal Protective Equipment: BAME HCWs are more likely to report lack of access, training and fit-testing regarding PPE compared to White counterparts.

A follow-up PPE survey of UK nursing staff (Royal College of Nursing; RCN, 2020) analysed responses from 4,418 HCWs: BAME $(n=782)$ and White British $(n=3,690)$. This survey found that BAME groups were underserved by PPE compared to White British peers in both non-high-risk 
environments and high-risk environments. Furthermore, $56 \%$ of BAME groups felt pressure to provide care for COVID-19 patients without adequate PPE compared to $29 \%$ of White British peers; while HCWs of BAME background were more likely to raise concerns about PPE (WB: 56\%; BAME: 67\%), they were more likely to have unaddressed concerns (WB:25\%; BAME 31\%). In line with this review's objective of frontline work, only data from high-risk environments shall be summarised.

BAME populations were significantly less likely to report availability of eye/face protection (WB: 66\%; BAME: 43\%) and filtering face piece protection (WB: 43\%; BAME: 25\%) than White British peers. Not only are BAME HCWs less likely than White British HCWs to initially access PPE, but they are also three times more likely to report not enough PPE for the duration of their shift: eye/face protection (WB: 8\%; BAME: 21\%); filtering masks (WB 15\%: BAME: $35 \%$ ); and for disposable fluid repellent gowns (WBL 19\%; BAME: 37\%). Since the initial survey, an even greater proportion of BAME workers report being asked to re-use single use PPE (53\%), compared to their White British peers $(42 \%)$.

Furthermore, White British frontline staff are 'considerably' more likely to receive PPE training - a third of BAME staff report not receiving essential training. PPE was also less likely to fit BAME respondents (46\%) compared to British respondents (38\%); yet only $49 \%$ of BAME respondents reported fit testing for filtering face piece compared to $74 \%$ of White British respondents. While access for fit testing had improved for White British respondents since the last survey from $68 \%$, the BAME situation has worsened from $53 \%$. Failed mask fitting tests has been suggested to stem from the fact that masks were modelled on White ethnicities (Ali, Adam, West, Pareek, Raza \& Iqbal, 2020). A recent qualitative study led by RREAL also pointed to issues with PPE fitting in women, as PPE sizes were more suitable for male bodies (Hoernke et al., 2021).

This is supported by a cross-sectional prospective survey ( $n=1119 ; 71 \%$ BAME) which found BAME respondents were significantly more likely to report concerns about PPE and believing this was contributing to HCW deaths than their white counterparts (Ali et al., 2020).

c. Afraid to speak up and a 'bully' culture: both viral exposure through re-deployment and access to PPE is thought to be worse for minoritized groups due to a lack of ability to say 'no', fearful of repercussions.

A cross-sectional prospective survey ( $n=1119 ; 71 \%$ BAME) found that BAME HCWs reported feeling less able to say ' $n o$ ' if asked to work without adequate PPE significantly more than their White counterparts; this was not linked with other factors such as gender, place of work, contract type, duration of employment or healthcare role (Ali et al., 2020).

In-depth interviews $(n=52)$ and a survey $(n=115)$ conducted with BME clinical representatives and stakeholders found that BME HCWs were more likely to report taking on high-risk roles and experience lack of PPE due to fear of speaking up about their experiences in case contracts would not be renewed, or that shifts would be reduced (Farah \& Saddler, 2020); this is particularly notable in instances where staff have vulnerable immigration status who fear having their Visa status challenged (Farah \& Saddler, 2020; Dean, 2020). Some 88\% of survey respondents reported that BME staff do not speak out because they fear losing their jobs. This may interact with fears of being labelled 'aggressive' or 'troublemakers' (Roye, 2020).

This is shocking when we consider the data by Cook et al. (2020) were individuals born outside of the UK were found to account for more than half of deaths while representing only $18 \%$ of the NHS workforce. This may stem from pre-existing systemic discrimination, bullying and harassment within the NHS (Otu et al., 2020). With this in mind, it is interesting that in a crosssectional survey of BAME HCWs in Leicester $(n=200)$ BAME HCWs defined PPE supply as 'adequate' and support as 'more than satisfactory' despite $31 \%$ of participants reporting issues securing PPE in their workplace and $58.5 \%$ perceiving lack of PPE as reasons for increased COVID19 death in BAME backgrounds (Moorthy \& Sankar, 2020).

\section{Emotional impact: BAME HCWs may experiences heightened and unique emotional strain}


a. Increased anxiety: BAME HCWs may experience anxiety on account of their increased mortality risk, a greater 'tear' between personal risk and sense of duty, and through the risk they may pose to family due to intergenerational living.

It is not hard to imagine that the increased risk faced by BAME nurses directly translates not only into physical health risk but also into fear, anxiety and psychological trauma (Moorley, Darbyshire, Serrant, Mohamed, Ali, \& De Souza, 2020; Otu et al., 2020). This is also contributed to feeling less supported and protected, a quarter of BAME staff reported not feeling confident in their employer's ability to protect them compared to 1 in 10 in White British staff; BAME staff are also less likely to have their concerns addressed compared to their White British counterparts (RCN, 2020).

This also presents BAME HCWs with a greater emotional dilemma in terms of trying to balance their moral duties and their need to look after their own health, 'stuck' between passion to help people and inflated personal risk (Shah et al., 2020). Moorthy and Sankar (2020) concluded from their survey of BAME HCWs $(n=200)$ that there was clear and urgent need to provide culturally competent mental health support for BAME healthcare workers. Another factor which may contribute to unique and inflated emotional drain on BAME HCWs is the increased likelihood that they reside in overcrowded, multigenerational households, which may place greater anxiety on passing COVID-19 family members (Otu et al., 2020). While this is a notable issue, consideration of BAME HCWs has yet to progress beyond their immediate physical health risk and literature on this remains sparse and colloquial. This has been pointed out as a critique of the PHE report on disparity (Shah et al., 2020).

b. Racial abuse: BAME HCWs experience racially motivated verbal abuse from patients/patient families, and, in some instances, even peers, which can drastically change their experience of providing care.

Beyond the systemic pressure and 'bully' culture alluded to previously in the report, BAME HCWs are also exposed to racially motivated abuse. This is not a new phenomenon within the NHS in light of COVID-19, but one which has gotten progressively worse since the political climate of Brexit which often hosts anti-immigration sentiment; some $40 \%$ of nurses report increased racism from patients and families following Brexit (Dangerfield, Ferrante, Jennings, \& Moorley, 2020). In the context of COVID-19 patient care, one commentary reflected the experience of a White nurse which commented on the gratitude she had received from a patient as she was being looked after by someone who was 'English', a remark made on account of all the other recovery nursers on the ward being of non-White ethnicity (Dangerfield et al., 2020). While such experiences may seem exceptional, they reflect a daily lived reality (Moorley et al. 2020; Brathwaite, 2018).

\section{CONCLUDING LITERATURE AND COMMENTS}

Unfortunately, the government response to the inequalities faced by BAME HCWs has been slow and underwhelming, as was perceived by the majority (81\%) of HCWs in the survey conducted by Ali et al. (2020). Interviewees in the study conducted by Farah and Saddler (2020) also felt that, in light of the broad evidence pointing to inequalities in experiences faced by BAME HCWs, the government has not taken enough action to address the underlying causes of these issues. Their survey revealed that 7 in 10 respondents felt that the introduced risk assessments for BAME staff were tokenistic, with many raising concerns about the impact this may have on future recruitment of BAME staff. It is felt by many that the PHE report missed the opportunity to consider sociocultural factors in inequalities, failing to truly investigate BAME HCW experiences compared to their White counterparts (Brathwaite, 2020). Furthermore, the lack of national tools and guidance to actually perform such risk assessments has resulted in severe delays in the implementation of the risk assessments, in addition to regional variation, which may have significant implications in risk stratification between hospitals and, 
ultimately, the protection of BAME HCWs; 'For some healthcare workers, these differences could be the difference between life and death.' (Abbas et al., 2020, p.1). There were also concerns that the report had been 'whitewashed' after comments from thousands of organisations and individuals appeared to be missing from the final report (lacobucci, 2020a). It later became apparent that a separate 64-page document was to be published which contained these comments and more practical recommendations, but many expressed deep concern that the government had not acknowledged the existence of the second report (lacobucci, 2020b).

To quote the RCN (2020, p.16): 'Too often, policy makers wait for certainty of causation before acting to resolve issues. In this case, they cannot wait for analysis and investigation into the wide range of factors which will have led to the disproportionate impact on BAME staff. There is already enough evidence to show that staff are being affected. Workplace experience and treatment is a likely factor contributing to the increased risk which BAME frontline staff are facing during this pandemic.' While much of the literature explored within this review stems from commentary style publications there is a clear wealth of evidence which exemplifies inequalities in HCWs' experiences of care provision during COVID-19. Steps should be taken not only to prioritise physical BAME HCWs' wellbeing but also their emotional and mental health needs (Vera San Juan et al. 2020). There is greater need for primary research, in particular qualitative data to narrate and capture the lived experiences of BAME HCWs during this pandemic.

\section{REFERENCES}

Abbas, A., Memon, S. F., Khattab, N., \& Abbas, A. R. (2020). COVID-19 risk assessments: shortcomings in the protection of Black, Asian and Minority Ethnic healthcare workers. The Journal of Hospital Infection, 106(2), 385-386.

Aldridge, R. W., Lewer, D., Katikireddi, S. V., Mathur, R., Pathak, N., Burns, R., ... \& Hayward, A. (2020). Black, Asian and Minority Ethnic groups in England are at increased risk of death from COVID-19: indirect standardisation of NHS mortality data. Wellcome open research, 5(88)1-20.

Ali, P., Adam, Z., West, J., Pareek, M., Raza, M., \& Iqbal, J. (2020). Perceptions of COVID-19-related risk and mortality among ethnically diverse healthcare professionals in the UK. Ethnicity \& Health, 110.

Brathwaite, B. (2018). Black, Asian and minority ethnic female nurses: colonialism, power and racism. British Journal of Nursing, 27(5), 254-258.

Chaudhry, F. B., Raza, S., Raja, K. Z., \& Ahmad, U. (2020). COVID 19 and BAME health care staff: Wrong place at the wrong time. Journal of Global Health, 10(2), 1-4.

Cook, T., Kursumovic, E., \& Lennane, S. (2020). Exclusive: deaths of NHS staff from covid-19 analysed. Health Serv J, 1-10.

Dangerfield, A., Ferrante, J., Jennings, K., \& Moorley, C. (2020). Practical action: The way forward to eliminating racially biased treatment of BAME nurses. Nursing in Critical Care, 25(6), 376-378.

Dean, E. (2020). How can we protect BAME nurses during the COVID-19 crisis?. Nursing Standard.

Farah, W., \& Saddler, J. (2020). Perspectives from the front line. https://www.nhsemployers.org//media/Confederation/Files/Publications/Documents/Perspectives-from-the-frontline_FNL_Dec2020.pdf

Ford, M. (2020). BME nurses "feel targeted" to work on Covid-19 wards. Nursing Times.

Hoernke, K., Djellouli, N., Andrews, L., Lewis-Jackson, S., Manby, L., Martin, S., ... \& Vindrola-Padros, C. (2021). Frontline healthcare workers' experiences with personal protective equipment during the COVID-19 pandemic in the UK: a rapid qualitative appraisal. BMJ open, 11(1), e046199. 
lacobucci, G. (2020a). Covid-19: PHE review has failed ethnic minorities, leaders tell BMJ. BMJ.

lacobucci, G. (2020b). Covid-19: Government will belatedly publish recommendations for protecting ethnic minorities. BMJ.

Intensive Care National Audit and Research Centre. (2020). ICNARC report on COVID-19 in critical care: 24 April 2020. https://naccs.org.uk/wp-content/uploads/2020/05/ICNARC-COVID-19-report-2020-0424.pdf

Moorley, C., Darbyshire, P., Serrant, L., Mohamed, J., Ali, P., \& De Souza, R. (2020). Dismantling structural racism: Nursing must not be caught on the wrong side of history. Journal of Advanced Nursing, 1-4.

Moorthy, A., \& Sankar, T. K. (2020). Emerging public health challenge in UK: perception and belief on increased COVID19 death among BAME healthcare workers. Journal of Public Health, 42(3), 486492.

NHS Workforce Race Equality Standard. NHS Workforce Race Equality Standard 2019 data analysis report for the NHS trusts. 2020. https://www.england.nhs.uk/wp-content/uploads/2020/01/wres2019-data-report.pdf

Office for National Statistics. (2020). Coronavirus (COVID-19) related deaths by ethnic group, England and Wales: 2 March 2020 to 10 April 2020.

https://www.ons.gov.uk/peoplepopulationandcommunity/birthsdeathsandmarriages/deaths/articles/cor onavirusrelateddeathsbyethnicgroupenglandandwales/2march2020to10april2020

Otu, A., Ahinkorah, B. O., Ameyaw, E. K., Seidu, A. A., \& Yaya, S. (2020). One country, two crises: what Covid-19 reveals about health inequalities among BAME communities in the United Kingdom and the sustainability of its health system?. International journal for equity in health, 19(1), 1-6.

Public Health England. (2020). Beyond the data: Understanding theimpact of COVID-19 on BAME groups.

https://assets.publishing.service.gov.uk/government/uploads/system/uploads/attachment_data/file/89 2376/COVID_stakeholder_engagement_synthesis_beyond_the_data.pdf

Regenold, N., \& Vindrola-Padros, C. (2021). Gender Matters: A Gender Analysis of Healthcare Workers' Experiences during the First COVID-19 Pandemic Peak in England. Social Sciences, 10(2), 43.

Royal College of Nursing. (2020). Second Personal Protective Equipment Survey of UK Nursing Staff Report: Use and availability of PPE during the COVID-19 pandemic.

https://www.rcn.org.uk/professional-development/publications/rcn-second-ppe-survey-covid-19pub009269

Roye, J. (2020). Are we doing enough for our BME workforce in nursing?. Practice Nursing, 31(9), 396397.

San Juan, N. V., Aceituno, D., Djellouli, N., Sumray, K., Regenold, N., Syversen, A., ... \& VindrolaPadros, C. (2021). Mental health and well-being of healthcare workers during the COVID-19 pandemic in the UK: contrasting guidelines with experiences in practice. BJPsych Open, 7(1).

Selvarajah, S., Abi Deivanayagam, T., Lasco, G., Scafe, S., White, A., Zembe-Mkabile, W., \& Devakumar, D. (2020). Categorisation and Minoritisation. BMJ Global Health, 5(12), 1-3.

Shah, N., Ahmed, I. M., \& Nazir, T. (2021). Torn Between Caution and Compassion: a Dilemma for Clinicians from Black and Minority Ethnic Groups During the COVID-19 Pandemic. Journal of racial and ethnic health disparities, 1-3.

Vindrola-Padros, C., Chisnall, G., Cooper, S., Dowrick, A., Djellouli, N., Symmons, S. M., ... \& Johnson, G. A. (2020a). Carrying out rapid qualitative research during a pandemic: emerging lessons from COVID-19. Qualitative health research, 30(14), 2192-2204. 
Vindrola-Padros, C., Andrews, L., Dowrick, A., Djellouli, N., Fillmore, H., Gonzalez, E. B., ... \& Johnson, G. (2020b). Perceptions and experiences of healthcare workers during the COVID-19 pandemic in the UK. BMJ open, 10(11), e040503.

Williamson, E., Walker, A. J., Bhaskaran, K., Bacon, S., Bates, C., Morton, C. E., ... \& Goldacre, B. (2020). OpenSAFELY: factors associated with COVID-19-related hospital death in the linked electronic health records of 17 million adult NHS patients. MedRxiv.

https://www.medrxiv.org/content/10.1101/2020.05.06.20092999v1 